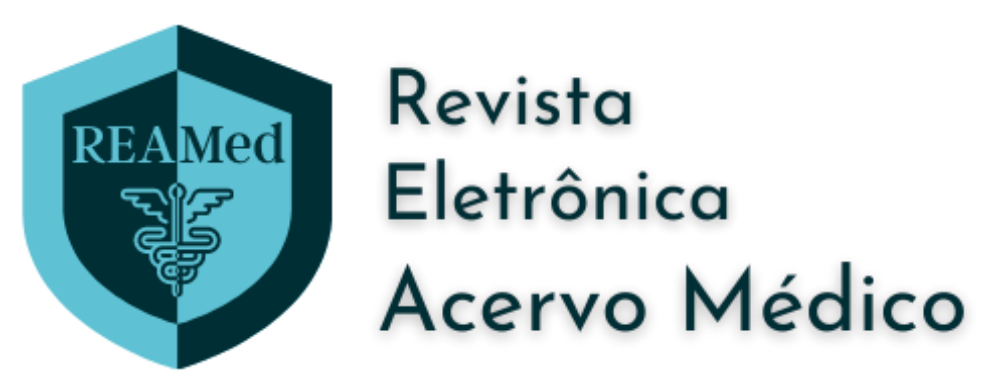

\title{
Abordagem geral da Doença Pulmonar Obstrutiva Crônica (DPOC): uma revisão narrativa
}

\author{
General approach to Chronic Obstructive Pulmonary Disease (COPD): a narrative review
}

Abordaje general de la Enfermedad Pulmonar Obstructiva Crónica (EPOC): una revisión narrativa

Arthur Emanuel Campos Coelho ${ }^{1 *}$, Carolina Izabela Santos Avelar ${ }^{1}$, Hallana de Lucena Araujo ${ }^{2}$, Isa Marcella Paiva Silva ${ }^{3}$, Leandra Neiva Jordão Mendes ${ }^{4}$, Julia de Oliveira Bernardino ${ }^{4}$, Luana Lohane Figueiredo de Aguilar ${ }^{4}$, Stephany Karen de Freitas Melo ${ }^{4}$, Yan Vasconcelos Carneiro², Suzana Tomaz Vasconcelos².

\section{RESUMO}

Objetivo: Relatar de forma atualizada uma abordagem geral da Doença Pulmonar Obstrutiva Crônica (DPOC). Revisão bibliográfica: A DPOC baseia-se em uma restrição progressiva do fluxo de ar pulmonar, causada por uma resposta inflamatória anormal. A consequência dessa inflamação é a redução da elasticidade pulmonar, dispneia, sibilância, muco, tosse, e hiperinsuflação pulmonar. A DPOC é definida como uma doença genética, porém quando há uma exposição por tabaco considerada, essa pode ser um fator determinante no processo saúde-doença. Além disso, a inalação prolongada da queima de biomassa poderá causar danos para a saúde do mesmo. O diagnóstico é feito na presença de fatores de risco para a doença, sinais e sintomas respiratórios crônicos e limitação persistente do fluxo de ar documentada por espirometria. Seu tratamento baseia-se na cessação imediata do tabagismo associado a fármacos que atuam no fluxo de ar. Considerações finais: A DPOC é uma pneumopatia incapacitante que repercute no cotidiano do paciente. Sua patogênese está ligada a hiperprodução de citocinas pró-inflamatórias que desencadeiam os sintomas típicos da DPOC, os quais são associados a exames complementares para diagnosticar e tratar esta enfermidade.

Palavras-chave: Doença pulmonar obstrutiva crônica, Obstrução, Abordagem.

\begin{abstract}
Objective: This study aims to report the updated approach to chronic obstructive pulmonary disease (COPD). Bibliographic review: COPD is based on the progressive restriction of pulmonary airflow caused by an abnormal inflammatory response. The consequence of that inflammation is reduced lung elasticity, dyspnea, wheezing, mucus, cough, and lung hyperinflation. Chronic Obstructive Pulmonary disease is defined as a genetic condition, but when tobacco exposure is considered, this can be a determining factor in the health-disease process. In addition, prolonged inhalation of biomass burning can cause health damage. Diagnosis is made in the presence of risk factors for the disease, chronic respiratory signs and symptoms,
\end{abstract}

1 Universidade de Itaúna (UIT), Itaúna - MG. *E-mail: arthur.emanuel.|@hotmail.com

2 Universidade de Fortaleza (UNIFOR), Fortaleza - CE.

${ }^{3}$ Centro Universitário Atenas (UniAtenas), Paracatu - MG.

${ }^{4}$ Centro Universitário Atenas (UniAtenas), Sete Lagoas - MG. 
and persistent airflow limitation documented by spirometry. The treatment is based on the immediate cessation of smoking associated with drugs that act directly on the patient's airflow. Final considerations: COPD is a disabling lung disease that affects primarily the patient's daily life. Its pathogenesis is linked to the overproduction of pro-inflammatory cytokines that triggers the typical symptoms of COPD, which are associated with complementary tests to diagnose and treat the disease.

Keywords: Chronic obstructive pulmonary disease, Obstruction, Approach.

\section{RESUMEN}

Objetivo: El estudio objetiva relatar un enfoque general actualizado de la enfermedad pulmonar obstructiva crónica (EPOC). Revisión bibliográfica: La EPOC es una restricción progresiva del flujo de aire pulmonar causada por una respuesta inflamatoria anormal. La consecuencia es una reducción de la elasticidad pulmonar, disnea, sibilancias, mucosidad, tos e hiperinsuflación pulmonar. La EPOC es una enfermedad genética, pero cuando hay una exposición al tabaco considerada por el paciente, puede ser un factor determinante en el proceso salud-enfermedad. Además, la inhalación prolongada de estos compuestos puede dañar su salud. El diagnóstico se realiza en presencia de factores de riesgo de la enfermedad, signos y síntomas respiratorios crónicos y limitación persistente del flujo aéreo documentada por espirometría. Su tratamiento se basa en la cesación inmediata del tabaquismo asociado a fármacos que actúan sobre el flujo aéreo. Consideraciones finales: La EPOC es una enfermedad pulmonar discapacitante que afecta la vida diaria del paciente. Su patogenia está relacionada con la sobreproducción de citocinas proinflamatorias que desencadenan los síntomas típicos de la EPOC, que se asocian a exámenes complementarios para diagnosticar y tratar esta enfermedad.

Palabras clave: Enfermedad pulmonar obstructiva crónica, Obstrucción, Abordaje.

\section{INTRODUÇÃO}

A Doença Pulmonar Obstrutiva Crônica (DPOC) se trata de uma enfermidade heterogênea que é caracterizada pela obstrução crônica do fluxo respiratório, agredindo especialmente os brônquios e/ou alvéolos e está associada a uma intensa resposta inflamatória. Sua prevalência global é de $5 \%$ a $13 \%$ e possui altos índices de mortalidade e morbidade; tendo como principal fator de risco o uso do tabaco (ROVERSI S, et al., 2016). Além do tabagismo, a exposição ao fumo, a poluição do ambiente, os gases irritantes/nocivos e a exposição ocupacional também corroboram para o desenvolvimento da patologia (SANTORO A, et al., 2019).

O mecanismo inflamatório consiste principalmente no acúmulo de neutrófilos e macrófagos encontrados na secreção pulmonar, expansão de citocinas pró-inflamatórias, sendo que o fator de necrose tumoral (TNF)- $\alpha$ aumenta com o envelhecimento bem como com a gradação da infecção (HUGHES MIJ, et al., 2020).

A DPOC está associada a sinais e sintomas de tosse, dispneia aos mínimos esforços, sibilância e expectoração crônica, os quais podem causar uma inflamação sistêmica que se manifesta em síndromes consumptivas como perda de peso e sarcopenia nas fases mais avançadas (VINIOL C e VOGELMIER FC, 2018). Por ser portador de uma doença crônica, o padrão clínico pode se alterar, caso ocorra um evento agudo (SANTORO A, et al., 2019).

O diagnóstico baseia-se principalmente na sintomatologia dos pacientes com tosse crônica com/sem escarro, sibilos e dispneia marcante. Cabe ressaltar que a espirometria é essencial para a análise clínica completa (ONISHI K, et al., 2017). Destaca-se ainda, tendências para ansiedade, depressão e até mesmo câncer de pulmão devido à proliferação celular, lesão celular endotelial pulmonar e neo-angiogênese induzida pela nicotina (GUT-GOBERT C, et al., 2019). Vários estudos demonstram que distúrbios cardíacos e vasculares crônicos, assim como a tuberculose são corriqueiros na DPOC, possivelmente devido aos coeficientes de risco em comum (ROVERSI S, et al., 2016).

O manejo terapêutico é bastante complexo, caracterizando-se essencialmente em precauções a fim de evitar a piora da função pulmonar, com decremento dos sintomas, especialmente pela cessação habitual do cigarro (SANTORO A, et al., 2019). Os principais tratamentos compõem-se de broncodilatadores, 
principalmente anticolinérgicos para o reparo e prevenção dos sintomas, adequando-se de maneira individualizada para cada paciente (ONISHI K, et al., 2017).

Esta revisão objetivou compactar as mais atualizadas fontes bibliográficas acerca da abordagem geral da DPOC, abrangendo sua fisiopatologia, quadro clínico/fatores de risco, avaliação diagnóstica, bem como seu manuseio terapêutico, por meio de artigos de ampla relevância clínica, com o fito de elucidar e sistematizar as informações que abrangem a doença pulmonar obstrutiva crônica.

\section{REVISÃO BIBLIOGRÁFICA}

A narrativa buscou na literatura demonstrar e esclarecer os diferentes aspectos e principais características da DPOC de maneira coesa e concisa. Sendo assim, para um melhor entendimento e clareza, o trabalho foi dividido em quadro subcategorias que tange a fisiopatologia, ao quadro clínico, ao diagnóstico e ao tratamento.

\section{Fisiopatologia}

A DPOC é uma restrição, geralmente, irreversível e progressiva do fluxo de ar no trato respiratório inferior causado por uma resposta inflamatória anormal dos pulmões a toxinas ou partículas inaladas, especialmente a fumaça do cigarro e alérgenos (MCCABE C, et al., 2017). Portanto, os fumantes devem ser aconselhados para a cessação do tabagismo, imediatamente (MORJARIA JB, et al., 2017).

A exposição dessas partículas por inalação pode causar inflamação das vias respiratórias e dos alvéolos. Este processo é mediado pelo aumento da atividade da protease e diminuição da atividade antiprotease liberada pelos neutrófilos e outras células inflamatórias. As proteases pulmonares dissolvem a elastina e o tecido conjuntivo durante o reparo normal do tecido. A atividade dessas proteases é geralmente equilibrada por anti-proteases, como a antitripsina $\alpha-1$. Esse desequilíbrio entre a protease e a anti-protease é o principal mecanismo do componente enfisema da doença, devido a deterioração da elastina e a subsequente perda de integridade alveolar, que consequentemente, causa hiperinsuflação e dificuldade de expiração (ARAÚJO D, et al., 2017).

A cicatrização e remodelação do trato aéreo leva a um aumento das camadas das vias aéreas com menos de $2 \mathrm{~mm}$ de diâmetro como: epitélio, lâmina própria, músculo liso e adventícia, desse modo, ocorre à perda constante de bronquíolos terminais (ARAÚJO D, et al., 2017).

Além da secreção excessiva de muco, broncoespasmo, fibrose peribrônquica e destruição das pequenas vias aéreas, a inflamação também pode causar um estreitamento e obstrução das vias aéreas. Isso geralmente ocorre devido à exposição prolongada a substâncias irritantes inaladas: queima de biomassa, tabagismo, alérgenos, pelos de animais e poeira, que induzem uma resposta inflamatória crônica nas pequenas vias aéreas, com presença de linfócitos e muco. Além disso, foram observados que muitos mediadores de processos inflamatórios sistêmicos estão relacionados com o surgimento de fraqueza de músculos esqueléticos e agravamento ou iniciação de problema cardíacos e metabólicos (VINIOL C e VOGELMEIER CF, 2018).

Infecções virais, bacterianas ou partículas irritantes ativam células imunes inatas, como células epiteliais, neutrófilos e macrófagos. Estas liberam moléculas e citocinas associadas ao constante estresse e danos sofridos, que acionam receptores de reconhecimento de padrões. Desse modo, é induzido padrões de respostas imunes adaptativas que incluem diversas células como: T (Th1 e Th17) CD4+, citotoxicidade CD8+ e respostas de células $B$ e plasmócitos, que desencadeiam a formação de agrupamentos linfóides na inflamação crônica (ONISHI K, et al., 2017). Comitantemente, foi observado uma alta contagem de eosinófilos no sangue de pacientes com frequentes exacerbações de DPOC (VINIOL C e VOGELMEIER CF, 2018).

Esse processo proliferativo constante causa um esforço excessivo de células caliciformes e hipertrofia da glândula submucosa, levando à secreção excessiva de muco, encurtamento e remodelamento das pequenas vias aéreas, a perda alveolar e alterações no leito vascular que levam a uma hipertensão 
pulmonar (ARAÚJO D, et al., 2017). Uma maior resistência das vias aéreas aumenta o trabalho e esforço respiratório e diminui o fluxo respiratório. A força expiratória depende muito da elasticidade pulmonar. Esta é prejudicada devido à degradação da elastina alveolar pelas proteases, caracterizando o enfisema pulmonar. É caracterizado como o fenômeno de estagnação do ar, que causa um aumento do volume aéreo residual, da capacidade funcional e da capacidade pulmonar totalitária (VINIOL C e VOGELMEIER CF, 2018).

\section{Quadro clínico}

A DPOC se caracteriza por uma redução da função pulmonar, visto que essa falta de ar na grande maioria das vezes piora com esforço físico, ademais a tosse crônica também é um sintoma frequente em diagnósticos comprovados de DPOC (BOUZA E, et al., 2020). Além disso, para ter a confirmação do diagnóstico de DPOC é necessário realizar exames laboratoriais complementares como a espirometria e a radiografia do tórax. Essa doença pulmonar, além de genética, ou seja, o próprio organismo do indivíduo pode ter uma predisposição a tê-la, ela também pode se relacionar com algumas exposições como o tabaco ou produtos derivados da biomassa (HUGHES MJ, et al., 2020). Apesar de o tabaco influenciar no desenvolvimento da DPOC, quase $40 \%$ dos indivíduos continuam fumando apesar de apresentarem os sintomas da mesma (BOUZA E, et al., 2020).

O quadro clínico de DPOC é particularmente caracterizado por uma resposta inflamatória exagerada, e esse processo inflamatório é predominantemente crônico que pode danificar o parênquima pulmonar, alterar brônquios e bronquíolos (BURKES RM e DRUMMOND MB, 2019). A consequência dessa deterioração é a redução da elasticidade pulmonar, sibilância, respiração com os lábios semicerrados, aumento excessivo de muco, utilização da musculatura acessória do pescoço, hiperinsuflação pulmonar que é definida pelo ar retido no pulmão, ou seja, não é esvaziado por completo e tosse (MORJARIA JB, et al., 2017).

Em casos mais avançados da DPOC, os pacientes se deparam em uma posição em que a sintomatologia é amenizada: sentada com inclinação do tórax para frente, com os braços esticados e apoiando o peso nas palmas das mãos. Uma das características da DPOC é uma doença exacerbada, e a definição dessa forma, constitui-se de uma dispneia acentuada com tosse produtiva e com aumento na expectoração purulenta (BOUZA E, et al., 2020).

O sintoma clássico da DPOC que leva os pacientes ao atendimento médico é a dispneia (SANTORO A, et al., 2019). Sendo assim, faz-se necessário a realização da anamnese e do exame físico de forma, extremamente cautelosa, quando se trata de pacientes profundamente sedentários, visto que, a escassez de exercícios físicos acarreta em uma ausência de dispneia devido a não realização de esforços físicos. Logo, esse sedentarismo prejudica a visualização desse sintoma, o que pode prejudicar ou retardar o diagnóstico de DPOC (ROVERSI S, et al., 2016). Portanto, a atividade física auxilia na exacerbação dos sintomas, uma vez que, os pacientes procuram atendimento médico apenas quando observam importante comprometimento da função pulmonar (BURKES RM e DRUMMOND MB, 2019).

Há variações de fatores de risco da DPOC, como o sexo, anormalidades genéticas, hormônios, desenvolvimento pulmonar anormal, maconha, vaped, cigarro, charutos, narguile entre outros (SANTORO $A$, et al., 2019). Os hormônios femininos influenciam negativamente em mulheres que têm uma predisposição para a doença pulmonar obstrutiva crônica, portanto, $35 \%$ das mulheres que têm o diagnóstico de DPOC e de osteoporose estão relacionadas com um maior grau de dispneia (BOUZA E, et al., 2020). Ademais, distúrbios como a depressão, insônia e ansiedade influenciam de maneira negativa nos sintomas, mas quando feito alguma atividade física, há uma progressão significativa e positiva no tratamento da doença (ROVERSI S, et al., 2016).

\section{Diagnóstico}

Hoje é amplamente reconhecido que a DPOC é uma síndrome heterogênea e complexa, com envolvimento pulmonar e extrapulmonar (ROCHE N, et al., 2018). É possível estabelecer um diagnóstico diferencial da DPOC em relação às outras doenças parenquimatosas pulmonares através de métodos de imagens do tórax como o raio-x, que nas outras doenças do parênquima pulmonar, se apresentam com um aumento nas marcações e características intersticiais. Além disso, existem alguns exames que analisam a 
aptidão e o funcionamento do pulmão que, nessas doenças exibem uma falha ventilatória restritiva ao invés de uma falha obstrutiva como observada na DPOC, contribuindo para a diferenciação das patologias (BURKES RM e DRUMMOND MB, 2019).

Atualmente na clínica, o diagnóstico de DPOC é feito com base na presença de fatores de risco para a doença, sinais e sintomas respiratórios crônicos (dispneia aos esforços, tosse com / sem expectoração) e na identificação de uma limitação persistente do fluxo de ar, documentado por espirometria após um teste broncodilatador (BOUZA E, et al., 2020). Os documentos atuais e a maioria das diretrizes recomendam o uso de espirometria simples para diagnosticar a DPOC e graduar sua gravidade. Essa abordagem simplista pode levar à classificação incorreta de vários indivíduos com DPOC por uma série de razões.

Uma vez que a DPOC tem seu diagnóstico confirmado nos primórdios de seu curso fisiopatológico, em associação simultânea com a erradicação dos seus fatores de risco, o comprometimento das funções pulmonares reduz consideravelmente, bem como contribuirá para uma recuperação mais efetiva e mais rápida dos pacientes (GUT-GOBERT C, et al., 2019).

Além disso, a razão fixa VEF1 / CVF <0,7 é um critério comumente usado na prática clínica para definir obstrução ao fluxo aéreo de acordo com a primeira recomendação GOLD e para diagnosticar DPOC. No entanto, apresentam alguns preconceitos de idade e sexo, o que pode levar a sobrediagnóstico em indivíduos mais velhos e homens, além de subdiagnóstico em indivíduos mais jovens e mulheres (ROGLIANI P, et al., 2017).

São vários achados e exames clínicos que levam ao diagnóstico de DPOC, não apenas a espirometria. A anamnese é um procedimento essencial, quando bem feito ela pode identificar, avaliar e verificar comorbidades, problemas nutricionais e sintomas psiquiátricos como depressão, bastante comuns nesses pacientes. Além disso, com essa pesquisa e história do paciente é possível estabelecer o perfil de risco de doenças cardíacas e vasculares (frequentemente alto nos pacientes com DPOC).

Outros exames clínicos também são opções, no entanto não são comuns como: os testes minuciosos de funcionamento pulmonar realizados em instituições e laboratórios especializados em fisiologia pulmonar que são capazes de analisar e de medir a difusão do monóxido de carbono (CDCO), curvas de fluxo-volume e a capacidade inspiratória (VINIOL C e VOGELMEIER CF, 2018). Na primeira consulta, preferencialmente, é solicitada a oxigenação arterial por oxímetro de dedo ou de pulso (este último com uma melhor precisão). Se a saturação de oxigênio for inferior a $90 \%$, a gasometria arterial precisa ser utilizada para avaliar e verificar a gravidade e as possíveis indicações de oxigenoterapia. O paciente observado deve ser reavaliado com base no estágio e número de ocorrências (PEREZ-PADILLA R e MENEZES AMB, 2019).

\section{Tratamento}

O tabagismo é o principal fator responsável pelo surgimento da Doença Pulmonar Obstrutiva Crônica DPOC, pois contribui diretamente para a fisiologia da doença, causando uma inflamação pulmonar, o que aumenta a produção de citocinas pró-inflamatórias, desencadeando os sintomas clássicos da enfermidade (SPIROPOULOU A, et al., 2019). Desse modo, a cessação do tabagismo é de extrema relevância para o controle da doença em pacientes fumantes que possuem DPOC, pois a não adesão ao tratamento e a não interrupção do uso crônico do cigarro poderá levar a uma deterioração mais rápida da função pulmonar e acarretar futuras complicações (SANTORO A, et al., 2019).

Dessa forma, a interrupção do tabagismo, que pode ser auxiliado por uma ampla gama de intervenções, dentre elas intervenções farmacológicas e psicossociais, comprovadas cientificamente para aumentar as taxas de sucesso do tratamento, auxiliam no não agravamento da DPOC (TIBERI S, et al., 2019). Ademais, é de extrema relevância reconhecer e intervir, precocemente, nos principais fatores de risco que contribuem para uma rápida perda da função pulmonar e complicações da doença, dentre esses fatores de risco podemos citar o baixo peso ao nascer, o tabagismo na infância, o tabagismo materno e a asma infantil, a identificação e o manejo rápido e precoce desses fatores contribuem para a lentificação do curso da doença, retardando o processo fisiopatológico e garantindo uma maior expectativa de vida para os pacientes que possuem essa enfermidade (BURKES RM e DRUMMOND MB, 2019). 
Terapias farmacológicas auxiliam no processo de lentificação da perda gradual da função pulmonar do paciente, dentre essas alternativas se destacam os broncodilatadores, que são extremamente úteis para a redução dos sintomas e para a redução da frequência das exacerbações agudas da patologia. Também, os broncodilatadores, beta2-agonistas de longa ação, juntamente com os anticolinérgicos, fazem parte das opções disponíveis para a abordagem terapêutica da DPOC (ONISHI K, et al., 2017).

Outro medicamento testado para o tratamento da DPOC leve, com exacerbações e poucos sintomas, foi o tiotrópio, porém os estudos realizados com esta medicação não foram conclusivos. Também, antagonistas muscarínicos em conjunto com a sustentação da cessação do tabagismo, melhoram os sintomas da DPOC. Outrossim, tratamentos alternativos estão sendo desenvolvidos e os avanços recentes na nanotecnologia e na pesquisa de células-tronco lançaram uma nova luz sobre o tratamento atual da doença crônica das vias aéreas (PASSI M, et al., 2020).

\section{CONSIDERAÇÕES FINAIS}

A DPOC é uma doença crônica e progressiva grave, com um curso de incapacitação que repercute diretamente na vida diária do paciente e está intimamente ligada ao aumento da produção crônica de citocinas pró-inflamatórias. Estas afetam o parênquima pulmonar e desencadeiam as manifestações clínicas típicas da enfermidade, como a dispneia, tosse crônica e a utilização de musculatura acessória, com eventuais episódios de exacerbação. Nesse sentido, esse trabalho buscou realizar um estudo abrangente sobre essa doença pulmonar bastante recorrente com o intuito de contribuir com a comunidade médica no diagnóstico, nas características clínicas e laboratoriais da DPOC, além de elucidar sobre tratamentos e melhores condutas a serem seguidas pelos profissionais da saúde.

\section{REFERÊNCIAS}

1. ARAÚJO D, et al. Asthma-chronic obstructive pulmonary disease overlap syndrome - Literature review and contributions towards a Portuguese consensus. Rev Port Pneumol., 2016; 1-10.

2. BOUZA E, et al. Chronic obstructive pulmonary disease (COPD) in Spain and the different aspects of its social impact: a multidisciplinary opinion document. Rev Esp Quimioter., 2020; 33(1): 49-67.

3. BURKES RM, DRUMMOND MB. Initiating drug therapy in early stage chronic obstructive pulmonary disease: does it impact the course and outcome?. Current Opinion in Pulmonary Medicine, 2019; 25(2): 132-137.

4. GUT-GOBERT C, et al. Women and COPD: do we need more evidence? European Respiratory Review, 2019; 28(1): 151-159.

5. HUGHES MIJ, et al. Mecanismos compartilhados de multimorbidade em DPOC, aterosclerose e diabetes tipo 2: 0 neutrófilo como um potencial alvo inflamatório. Revisão Respiratória Europeia, 2020; 28(1): 151-162.

6. MCCABE C, et al. Computer and mobile technology interventions for self-management in chronic obstructive pulmonary disease. Cochrane Library, 2017; 5(5): 1-36.

7. MORJARIA J, et al. E-cigarettes in patients with COPD: current perspectives. Int J Chron Obstruct Pulmon Dis, Online, 2017; 12(1): 3203-3210.

8. ONISHI K, et al. Total management of chronic obstructive pulmonary disease (COPD) as an independent risk factor for cardiovascular disease. Journal of cardiology, 2017; 70(2): 128-134.

9. PASSI M, et al. Conventional and Nanotechnology Based Approaches to Combat Chronic Obstructive Pulmonary Disease: Implications for Chronic Airway Diseases. Int J Nanomedicine, 2020; 15(11): 3803-3826.

10. PEREZ-PADILLA R, MENEZES AMB. Chronic Obstructive Pulmonary Disease in Latin America. Annals of Global Health, 2019; 85(1): 7-15.

11. ROCHE N, et al. Personalised COPD care: Where are we going?. Revue de pneumologie Clinique, 2018; 74(5): 315326.

12. ROVERSI S, et al. Chronic Obstructive Pulmonary Disease and Cardiac Diseases. An Urgent Need for Integrated Care. Am J Respir Crit Care Med., 2016; 194(11): 1319-1336.

13. ROGLIANI P, et al. Multidimensional approach for the proper management of a complex chronic patient with chronic obstructive pulmonary disease. Expert review of respiratory medicine, 2018; 12(2):103-112.

14. SANTORO A, et al. Tobacco Smoking: Risk to Develop Addiction, Chronic Obstructive Pulmonary Disease, and Lung Cancer. Recent Patents on Anti-Cancer Drug Discovery, 2019; 14(1): 39-52.

15. SPIROPOULOU A, et al. Revisão da associação entre periodontite e doença pulmonar obstrutiva crônica em fumantes. Arquivo Monaldi para Doença torácica, 2019; 89(185).

16. TIBERI S, et al. Manejo da tuberculose grave e suas sequelas: da uti à cirurgia e reabilitação. Jornal brasileiro de pneumologia, 2019; 45(2).

17. VINIOL C, VOGELMEIER CF. Exacerbations of COPD. European Respiratory Review, 2018; 7(147). 